\title{
LE CONTRÔLE SENSORI-MOTEUR CÉRÉBELLEUX
}

Mai 1999

\author{
Sélim ESKIIZMIRLILER ${ }^{1}$, Nathalie FORESTIER $^{1}$, Bertrand TONDU $^{2}$ et Christian \\ DARLOT $^{1}$
}

1 : Département de Traitement des Signaux et des Images, École Nationale Supérieure des Télécommunications, URA CNRS 820, Paris

2 : Département de Génie Informatique et Électrique, Institut National des Sciences Appliquées, Toulouse

\section{Introduction}

Le mouvement d'un membre peut être décrit par une fonction de plusieurs variables : forces, inertie, viscoélasticité, raideur etc. Pour piloter les mouvements rapides, plus brefs que les délais de retour et les durées de traitement des informations sensorielles, il faut que le Système Nerveux Central prenne en compte cette fonction biomécanique et en estime la fonction dynamique inverse $[1,2,3]$. Selon le principe que nous proposons, c'est en calculant des fonctions inverses approximées des fonctions directes des organes sensoriels et des membres que les voies cérébelleuses contribuent à la précision de la perception et du contrôle des mouvements. En effet, anticiper les effets des signaux moteurs serait la fonction propre du Cortex Cérébelleux dans le contrôle des mouvements, et c'est parce qu'il serait placé dans une boucle de rétroaction que le signal anticipatif calculé permettrait d'estimer une fonction inverse. Un modèle cybernétique du contrôle d'un segment de membre a permis de simuler cette inversion [4,5]. Or sa structure, qui résulte de considérations fonctionnelles, est comparable aux connexions anatomiques des voies cérébelleuses.

Pour étudier plus précisément la fonction des voies cérébelleuses, les éléments de ce modèle cybernétique (filtres passe-bande, sommateurs, dérivateurs etc.) sont maintenant remplacés par des réseaux de neurones formels adaptatifs, dont la connectivité est conforme à celle des réseaux de neurones réels, par exemple à la connectivité bien connue du Cortex Cérébelleux [6,7]. Le circuit de contrôle ainsi construit permet de piloter un segment de bras de robot actionné par deux muscles pneumatiques de McKibben.

\section{Modèle du contrôle moteur}

\subsection{Modèle cybernétique}

La figure 1 montre le modèle cybernétique. Les éléments $g_{G}$ et $g_{D}$ représentent les fonctions biomécaniques de deux muscles antagonistes agissant sur un segment de membre, et $G$ représente la dynamique du segment. Ces fonctions sont en première approximation modélisées par des filtres passe-bas dans le domaine de Laplace. Selon l'interprétation anatomique proposée, les éléments entourés par des lignes tiretées représenteraient le Cortex Cérébelleux, qui apprendrait des fonctions approximées $g_{G} *, g_{D} *$ et $G^{*}$ de ces fonctions biomécaniques, sous la supervision d'un signal professeur issu de l'Olive Inférieure. 
L'Olive Inférieure détecte les erreurs dans les mouvements (pointage ou vitesse insuffisants ou excessifs) et envoie par les Fibres grimpantes (en pointillé sur la figure), des signaux qui corrigent les mouvements en cours, régulent les fonctions du Cortex et des Noyaux Cérébelleux, et déterminent ainsi le gain des réactions sensori-motrices.

\subsection{Introduction de réseaux de neurones formels dans ce modèle}

Les éléments qui représentent le Cortex Cérébelleux sont maintenant remplacés par des réseaux de neurones formels dont l'architecture est directement inspirée de la connectivité anatomique (figure 2). Chaque cercle grisé symbolise un neurone formel caractérisé par deux fonctions non linéaires : une fonction de recrutement en entrée (décrite par une équation différentielle) et une fonction sigmoïde en sortie (assurant une saturation des réponses). Chaque neurone formel représente le fonctionnement de tout un groupe de neurones réels. Conformément aux principales caractéristiques anatomiques du Cortex Cérébelleux, la connectivité du réseau présente :

- Une grande divergence des informations apportées par les fibres moussues aux cellules granulaires,

- Des boucles de rétroaction Golgi/grains qui participent au traitement des signaux afférents,

- Une grande convergence des signaux issus des grains (et transmis le long des fibres parallèles) au niveau des arborisations dendritiques des cellules de Purkinje.

\subsection{Règle d'apprentissage}

Les signaux de sortie du réseau de neurones formels déterministes qui modélise le Cortex Cérébelleux sont des ordres prémoteurs. Or les décours temporels que ces ordres doivent présenter pour que les mouvements soient correctement effectués ne sont pas connus. Un apprentissage supervisé classique ne peut donc pas être utilisé pour régler les poids synaptiques dans ce réseau, et c'est pourquoi nous avons choisi de les régler au moyen d'un apprentissage par renforcement séquentiel semi-supervisé.

Plusieurs sites d'apprentissage existent : toutes les synapses proches d'une terminaison d'une collatérale de fibre grimpante sont vraisemblablement adaptables. Des simulations ont ainsi montré que des modifications d'efficacité synaptique entre les fibres moussues et les dendrites des cellules granulaires, dans les glomérules, pourraient permettre de choisir les signaux pertinents pour combiner des informations sensorielles. Ces modifications sont induites par les signaux des fibres grimpantes qui parviennent aux glomérules par les cellules de Golgi. Mais pour piloter un segment de membre, l'apprentissage à la jonction des fibres parallèles et des cellules de Purkinje suffit.

Pour former le signal professeur, les positions voules et atteintes du segment mobile sont comparées et quand leur différence excède un seuil, un signal est envoyé vers le réseau de neurones. Ce signal est une simple impulsion d'amplitude fixe, qui dure 5 pas de temps. Elle ne code donc pas l'amplitude de l'erreur constatée. Comme le mouvement est déjà fini quand le signal professeur atteint les dendrites des cellules de Purkinje, l'activité dans le réseau de neurones n'est plus celle qui a permis de commander le mouvement. Pour déterminer quelles synapses doivent être adaptées, une éligibilité est calculée pour chacune conformément à l'équation (1).

$$
T_{i}=\frac{d e_{i}}{d t}+e_{i}(t)=g r_{i}(t)
$$

Une éligibilité peut être calculée au moyen de n'importe quelle fonction telle que sa valeur ait comme propriétés :

- d'être nulle avant que la synapse ne soit active, 
- d'augmenter quand la synapse est active,

- de décroître assez lentement pour n'être pas nulle quand le signal professeur arrive.

La variation d'efficacité synaptique est alors proportionnelle au produit de l'éligibilité et du signal professeur. Le règle d'apprentissage devient alors:

$$
\begin{aligned}
\Delta w_{g r \rightarrow P}(i) & =-\alpha \cdot F G(t) \cdot e_{i}(t) \\
\text { où } \quad \mathrm{FG}(\mathrm{t}) & =\text { signal professeur } \\
\alpha & =\text { taux d' apprentissage }
\end{aligned}
$$

Pendant l'apprentissage, un ensemble de 10 mouvements est répété jusqu'à ce que l'écart moyen (racine carrée de l'erreur quandratique moyenne) entre le mouvement voulu et le mouvement accompli soit inférieur à 1 degré.

\section{Simulations et résultats expérimentaux}

Le modèle tout entier, comportant un réseau de neurones inclus dans le circuit cybernétique initial, a été appliqué au contrôle d'un bras de robot mû par deux muscles pneumatiques de McKibben. Le site expérimental et son schéma de montage sont montrés dans la figure 3. Le système périphérique peut être décrit par les équations suivantes [8]:

$$
\begin{aligned}
& \left\{\begin{array}{l}
J \frac{d^{2} \theta}{d t^{2}}=R\left[F_{1}(t)-F_{2}(t)\right] \\
F_{i}(t)=F_{\max }\left(P_{0}+\Delta P_{i}\right)\left[1-\frac{1}{\varepsilon_{m}}\left(\varepsilon_{0}+\frac{R \theta}{l_{0}}\right)\right]-C \frac{R}{l_{0}} \frac{d \theta}{d t}
\end{array}\right. \\
& \text { où } \quad \mathrm{J} \quad=\text { Moment d' inertie du bras }=0.05 \mathrm{~kg} / \mathrm{m}^{2} \\
& \mathrm{C} \quad=\text { Coéfficient visqueuse }=100 \mathrm{~N} . \mathrm{s} \\
& \mathrm{R} \quad=0.015 \mathrm{~m} ; \mathrm{P}_{0}=2.5 \text { bars } ; 1_{0}=0.3 \mathrm{~m} ; \\
& \mathrm{F}_{\max }=20 \mathrm{kgf} ; \quad \varepsilon_{0}=0.1 ; \varepsilon_{\mathrm{m}}=0.25
\end{aligned}
$$

Le signal d'entrée du modèle est le profil de vitesse du mouvement voulu. Les ordres moteurs sont alors les variations de pression dans les muscles, que le fonctionnement du modèle tout entier régule selon la vitesse voulue et la position finale visée, en tenant compte des caractéristiques mécaniques du bras. L'apprentissage a d'abord été simulé en remplaçant les fonctions g et $\mathrm{G}$ par celles, connues, des muscles pneumatiques et du membre, puis il a été effectué sur le robot même, en itérant 300 fois un ensemble de 10 mouvements différents. Les délais moteurs et les délais de transmission dans les voies cérébelleuses ont été fixés respectivement entre $20-50$ et 1-10 ms.

Les résultats apparaissent sur la figure 4 où les lignes continues et tiretées représentent respectivement les mouvements voulus et accomplis. La figure 4A et 4B montre respectivement la performance d'apprentissage en simulation et en expérimentation, effectué sur le site montré dans la figure 3, en réponse aux mêmes ordres moteurs. Un ensemble de mouvements différent de celui utilisé pour l'apprentissage a été aussi testé, et les résultats apparaissent sur la figure 4c. Dans les deux cas les performances obtenues ont été comparables. 


\section{Conclusion}

Le modèle proposé permet de contrôler précisément un bras de robot mû par deux muscles artificiels, et les résultats des simulations et des expérimentations sont en très bon accord. La grande divergence d'information à l'étage d'entrée du réseau de neurones fournit des signaux nombreux et divers, traités dans les boucles de rétroaction Golgi/grain, qui comportent des gains et des délais. Ces signaux parviennent ensuite aux cellules de Purkinje, qui forment la sortie du réseau, où ils sont combinés au niveau des synapses entre les fibres parallèles et les cellules de Purkinje. Les poids des connexions sont régulés par un signal d'erreur qui code le sens de l'erreur mais pas son amplitude. Un réseau de neurones dont la connectivité reproduit celle du Cortex Cérébelleux peut ainsi apprendre des fonctions complexes au moyen de règles de fonctionnement et de régulation très simples.

Insérer un réseau de neurones formels reproduisant la connectivité neuronale dans un circuit cybernétique basé sur des principes fonctionnels permet donc à la fois de piloter un membre artificiel et de reproduire des observations physiologiques.

\section{Références}

[1] J. S. Albus. A theory of cerebellar function. Mathematical Biosciences, 10 :25-61, 1971.

[2] J. C. Eccles, M. Ito and J. Szentagothai. The cerebellum as a neuronal machine. Springer, 1967.

[3] D. Marr. A theory of cerebellar cortex. Journal of Physiology, 202 :437-470, 1969.

[4] L. Zupan. Modélisation du Réflexe Verstibulo-Oculaire et Prédiction des Cinétoses. Thèse de doctorat, Ecole Nationale Supérieure des Télécommunications, Janv. 1995.

[5] C. Darlot, L. Zupan, O. Etard and A. Maruani. Computation of inverse dynamics for the control of movements. Biological Cybernetics, 75 :173-186, 1996.

[6] N. Schweighofer, M. A. Arbib aand P. F. Dominey. A model ofthe cerebellum in adaptive control on saccadic gain. I- the model and its biological substrate, Biological Cybernetics, 75 :19-28, 1996.

[7] N. Schweighofer, M. A. Arbib and P. F. Dominey. A model of the cerebellum in adaptive control on saccadic gain. II- simulation results, 75 :29-36, 1996.

[8] B. Tondu et P. Lopez. Theory of an artificial pneumatic muscle and application to the modeling of McKibben artificial muscles. C.R.A.S., t. 320 :105-114, 1995. 


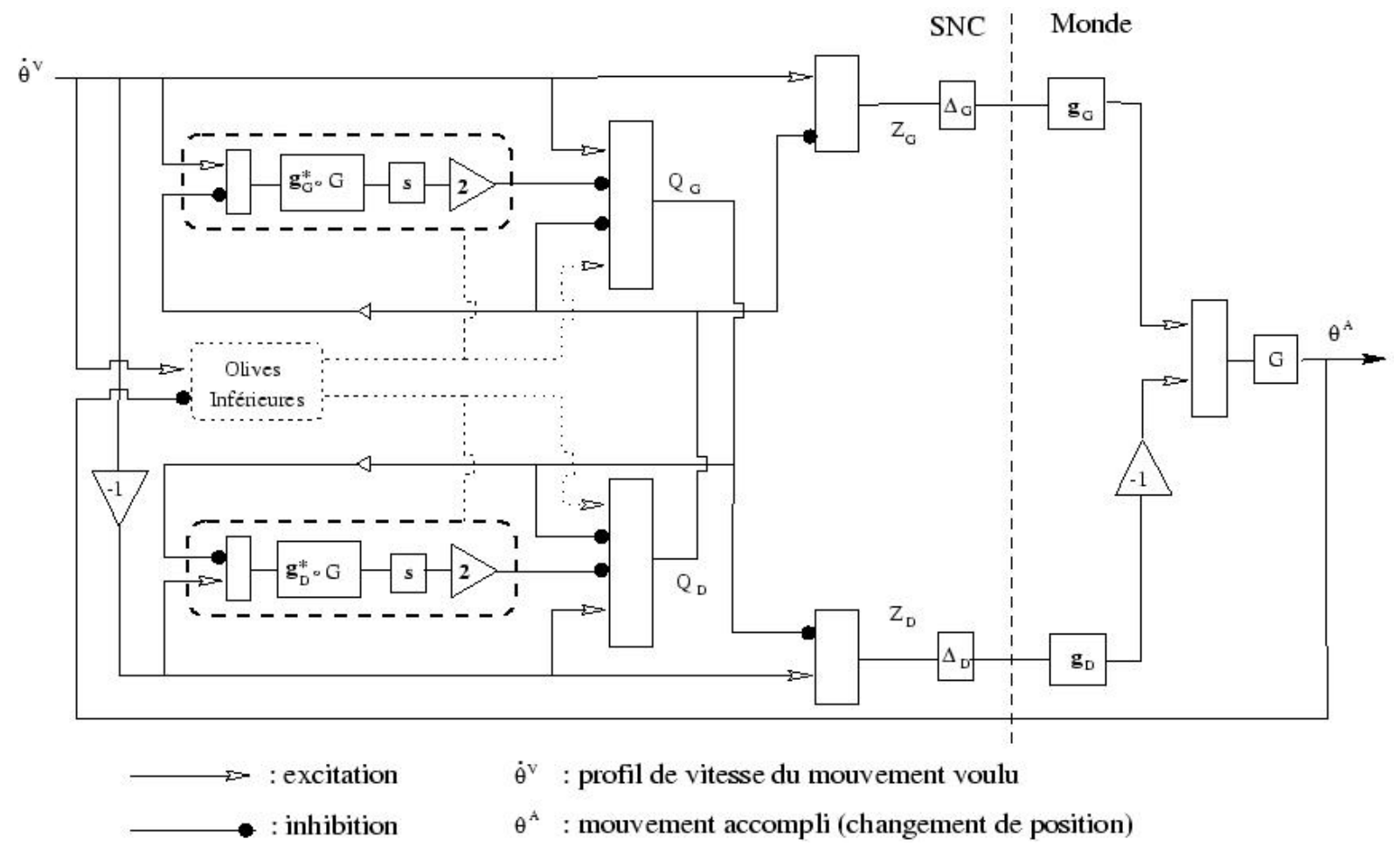

Figure 1: Modèle cybernétique initial

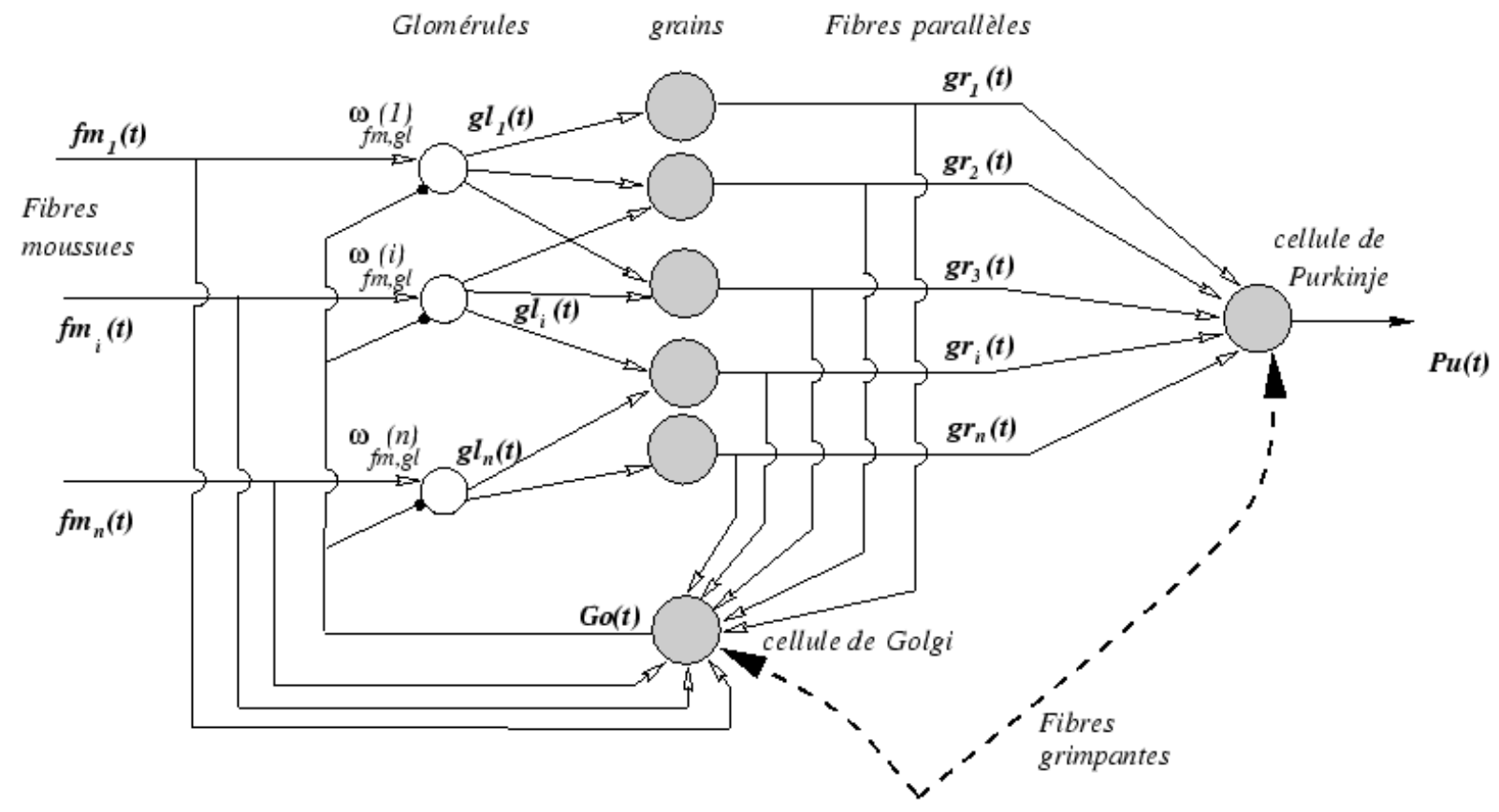

Figure 2: Architecture du réseau de neurones utilisé 


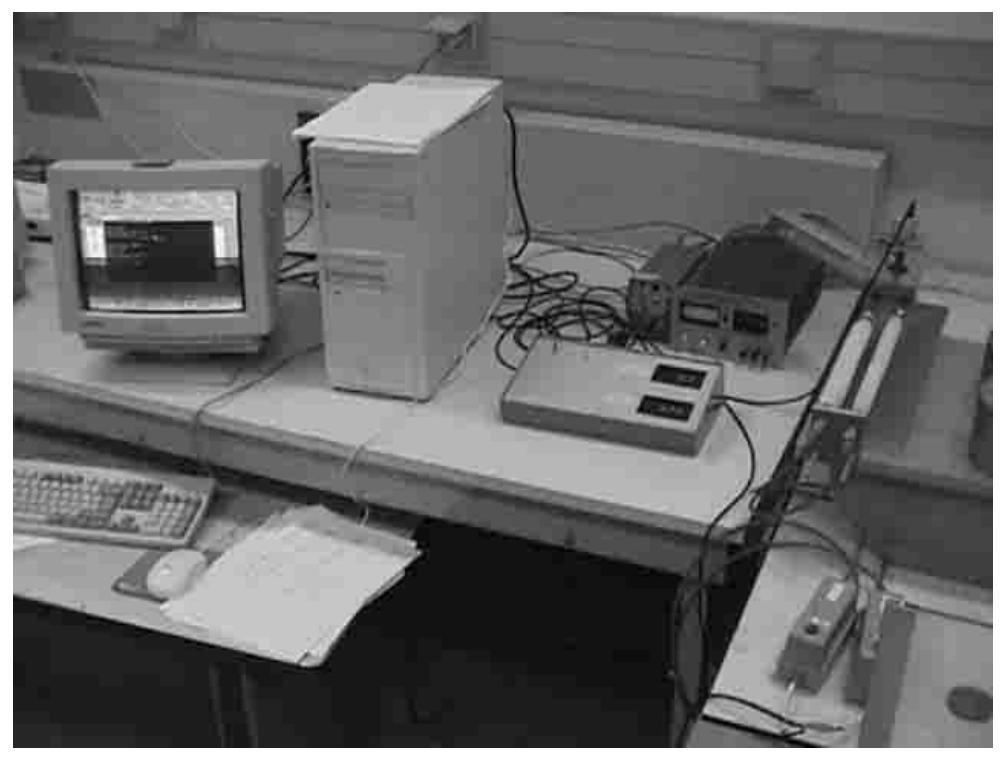

A)

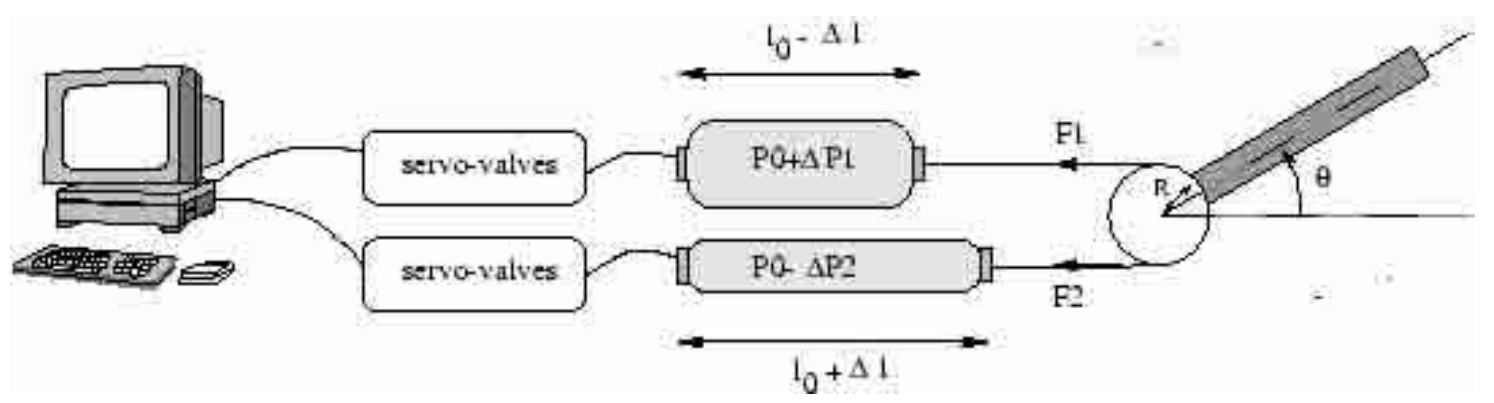

(B)

Figure 3: A) Le site expérimental avec deux muscle de McKibben. B) La représentation schématique du contrôle d'un bras mu par des muscles de McKibben.

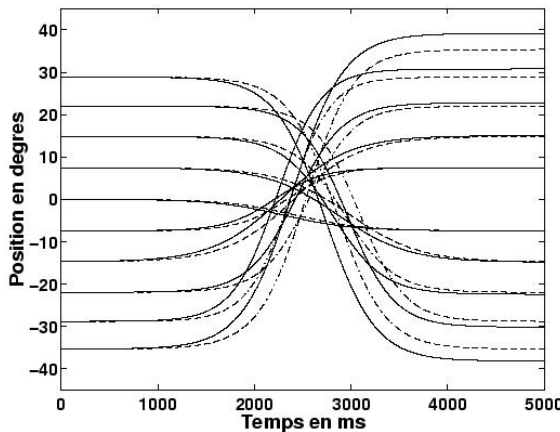

(A)

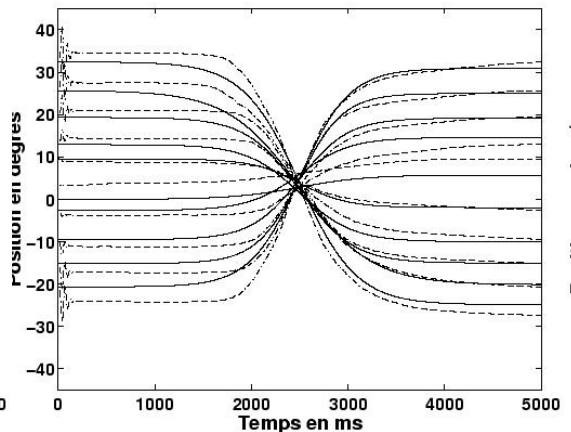

(B)

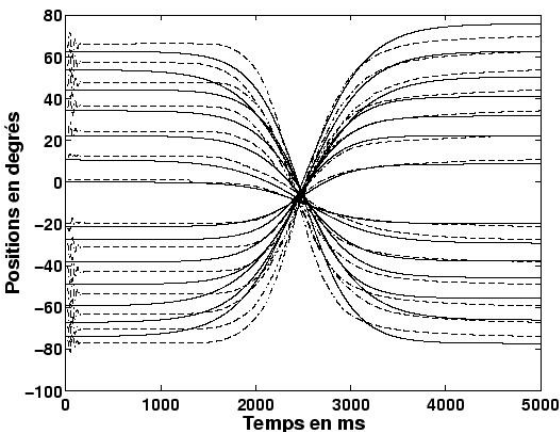

(C)

Figure 4: Performance du système après l'apprentissage A) Performance d'apprentissage en simulation B) Performance d'apprentissage en expérimentation C) Performance du robot sur les mouvements non appris. 